\title{
Review of variations in origin of left circumflex coronary artery
}

\author{
P. R. Roy, ${ }^{1,2}$ A. Saunders, and G. E. Sowton \\ From the Cardiac Department, Guy's Hospital, London
}

During the performance of 200 routine coronary angiograms, 5 patients with anatomical variants of the origin of the left circumflex coronary artery were found. The origin and course of the left circumflex coronary artery is described in each case.

The practical importance of these variant vessels in patients being considered for coronary artery surgery is emphasized.

Advances in coronary arterial surgery haveled directly to the routine use of angiographic visualization of the coronary arteries. The normal anatomy of the coronary arteries has been described in detail (James, 1961; Sewell, 1966). Anatomical variations of the coronary arteries are not uncommon, but the prevalence of these variations is hard to evaluate as there are few prospective studies available. Their recognition, particularly in the patient presenting with angina, is important, as decisions with regard to revascularization procedures depend on a knowledge of the anatomy of each individual artery.

That variations occur has long been known and the German anatomist Thebesius described the single coronary artery in 1716 . More recent comprehensive descriptions have been made by Edwards (I958).

Ogden and Stansel (1971) have previously classified the congenital variations of the coronary artery tree into three main types.

I) Variations in the origin of the vessel from the aorta, the major elements of the distal distribution being normal.

2) Major abnormalities in which there is either a communication between a coronary artery and a cardiac chamber (arteriovenous fistula) or an anomalous origin of the proximal coronary artery from the pulmonary artery rather than from the aorta.

3) Secondary variations representing a circulatory response to the primary intracardiac pathological defect.

Received 15 August 1974.

I Supported by a grant from the Postgraduate Medical Committee of the University of Sydney.

2 Present address: National Heart Hospital, Westmoreland Street, London WI.
It is our purpose in particular to illustrate the variations of the left circumflex artery and to describe the other variations that can be classified in type I above.

\section{Subjects and methods}

The five cases described were encountered during the performance of 200 coronary angiograms at Guy's Hospital between March 1972 and March 1974. In 2 of these 5 patients coronary bypass surgery was successfully undertaken on the atherosclerotic variant vessel.

\section{Case I}

A 47-year-old man with mitral regurgitation complained of chest pain suggestive of angina. He was catheterized with a view to mitral valve replacement. Coronary angiography was performed to exclude coronary artery disease as a major cause of his symptoms. During injection of contrast material into the left coronary ostium the left anterior descending coronary artery alone appeared to fill, suggesting occlusion of the circumflex artery (Fig. I). However, a slight amount of contrast could be seen passing into the left circumflex artery and slight manipulation of the catheter produced opacification of the circumflex artery (Fig. 2). There was in effect no main left coronary artery. Haemodynamic findings were consistent with mild mitral regurgitation and the patient was subsequently managed on medical therapy.

\section{Case 2}

A 62-year-old man presented with angina refractory to medical treatment. Coronary angiography with a view to saphenous vein bypass grafting was undertaken. During initial injection of the left main coronary artery no circumflex branch could be seen (Fig. 3). After injection of the right coronary artery, when no abnormal vessel or anastomosis to the area of the circumflex artery was seen, 

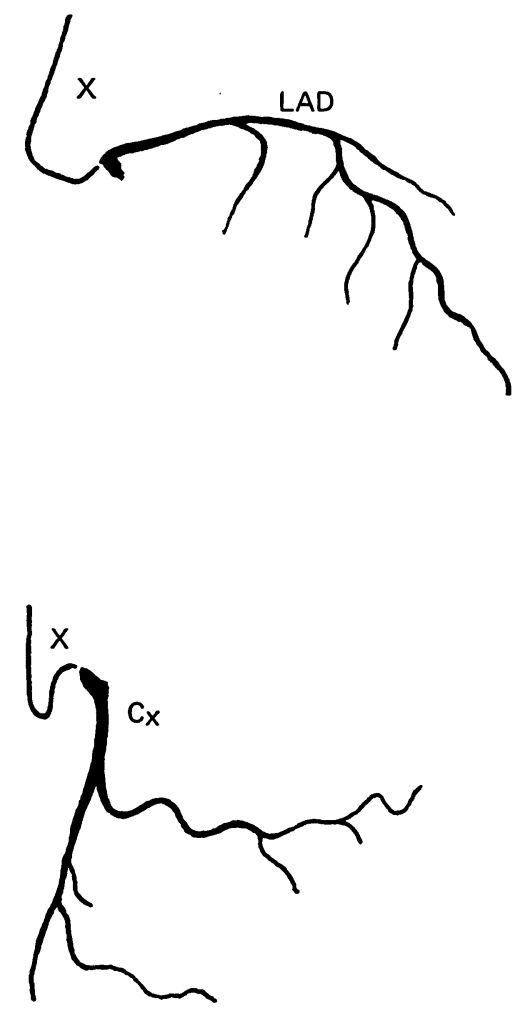

(2)
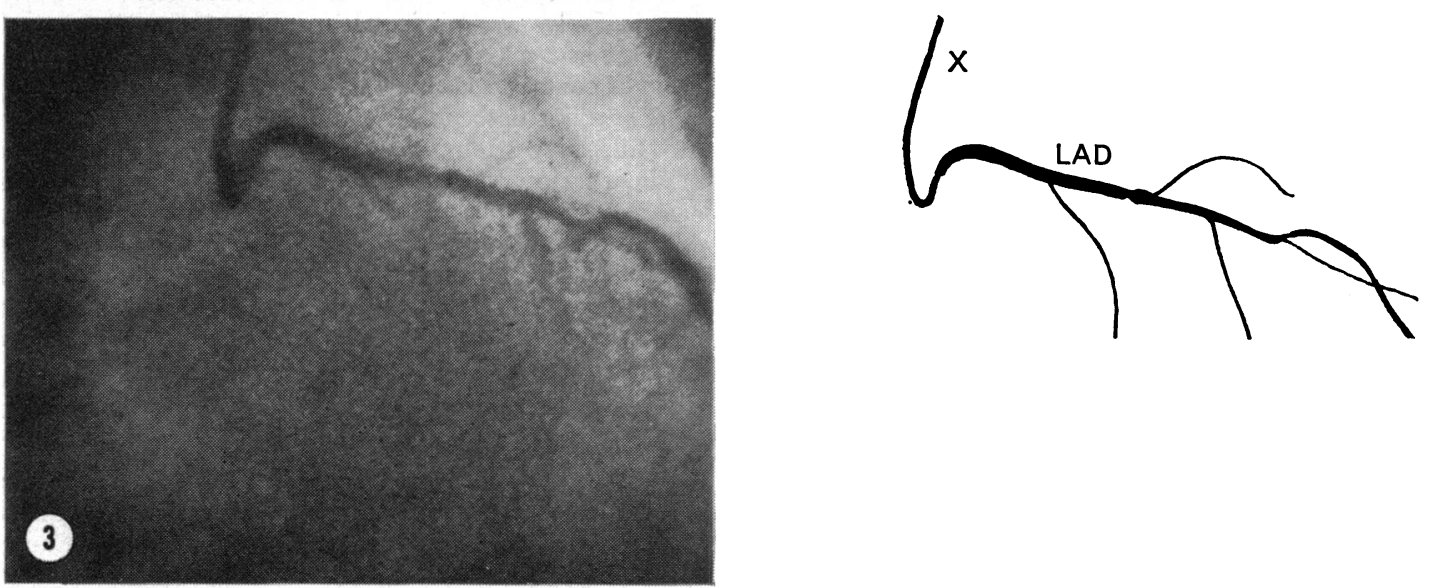
further attempts were then made to visualize the circumflex coronary artery. A separate ostium was found adjacent to the left main coronary ostium and the vessel was visualized (Fig. 4). Subsequently the patient underwent successful saphenous vein bypass grafting to both the variant circumflex coronary artery and the left anterior descending coronary artery. A surgically correctable lesion would not have been found without the selective demonstration of the variant circumflex vessel.

\section{Case 3}

The patient was a 60-year-old man who presented with frequent episodes of severe palpitations associated with central chest pain. There was some doubt about the cause of his pain, which had caused him to stop working, and coronary angiography was performed. The main left and the left anterior descending coronary arteries appeared normal. The circumflex artery was not visualized on the left side. Following injection of the right coronary artery which also appeared normal, further attempts to visualize the left circumflex artery were made. A vessel with a separate ostium close to that of the right coronary artery was found. This vessel passed across from the right coronary sinus around the aorta to supply the area normally supplied by the left circumflex artery (Fig. 5). There was no evidence of significant coronary artery disease and the chest pain disappeared with reassurance. Intermittent atrial fibrillation was found to be the cause of his episodes of palpitations and this was controlled with digoxin and propranolol.

\section{Case 4}

The patient was a 52-year-old woman with mitral valve disease and angina. The findings at coronary angiography were similar to those in Case 3. Normal left anterior descending and right coronary arteries were visualized. When the right coronary artery was selected for a second injection, slight manipulation of the catheter revealed the circumflex artery arising from the origin of the right coronary artery. This vessel (as in Case 3) passed around the aorta to supply the area normally supplied by the left circumflex artery (Fig. 6). The patient was subsequently managed with medical therapy.

\section{Case 5}

A 53-year-old man with angina underwent coronary arteriography at another hospital with a view to saphenous vein bypass surgery. He appeared to have a total occlu- sion of the left main coronary artery. On review the vessels could be seen passing from the right coronary artery across to the left side of the heart. A second coronary angiogram at Guy's was performed and a careful search was made for the left coronary artery. No vessel was found and the blood supply of the whole left side of the heart came from three anomalous vessels arising from the proximal right coronary artery. The pattern of blood supply is illustrated diagrammatically in Fig. 7. A saphenous vein graft to the right coronary artery was performed and at operation the anatomical appearances of the angiogram were confirmed. He has remained symptom free after operation.

\section{Discussion}

Multiple coronary ostia are probably the most common variations of the normal coronary vasculature. The right coronary artery not infrequently has two aortic ostia, the second being the ostium of the conus branch which passes in a semicircle around the pulmonary artery to anastomose with the main left or left anterior descending coronary artery. Schlesinger, Zoll, and Wessler (1949) found a separate origin of the conus artery in 50 per cent of patients. Zumbo et al. (1965) in a study of 2000 necropsies found a separate conus branch in only to per cent. This artery may be a major source of collateral flow when there is obstruction of the proximal portions of the right coronary artery or left anterior descending branch. A very hypoplastic right coronary artery extending a short distance from the aorta is occasionally seen, with the major distal circulation arising from the left coronary artery. Two other rare variations in the origin of the right coronary artery are, first, the single coronary artery arising from the left coronary sinus, and, secondly, the absence of a coronary ostium on the right with both coronary ostia arising from the left coronary sinus. Ogden and Goodyer (1970) have described the patterns of a single coronary artery in detail.

The left coronary artery is said virtually always to arise from a single ostium (James, 196I). The position of the ostium can vary from the cusp margin to a position I to $2 \mathrm{~cm}$ above the sinus margin. Both

FIG. I (Case I). Right anterior oblique projection showing the left anterior descending artery $(L A D)$. There is no main left coronary artery. The left anterior descending and left circumflex arteries arise together directly from the aorta. Note slight filling of the circumflex. In this and subsequent figures $X$ in the diagram identifies the injecting catheter.

FIG. 2 (Case I). Right anterior oblique projection showing the injection into the left circumflex artery $(C X)$.

FIG. 3 (Case 2). Right anterior oblique projection showing the left anterior descending artery $(L A D)$ arising directly from the aorta. There is a proximal stenosis. 

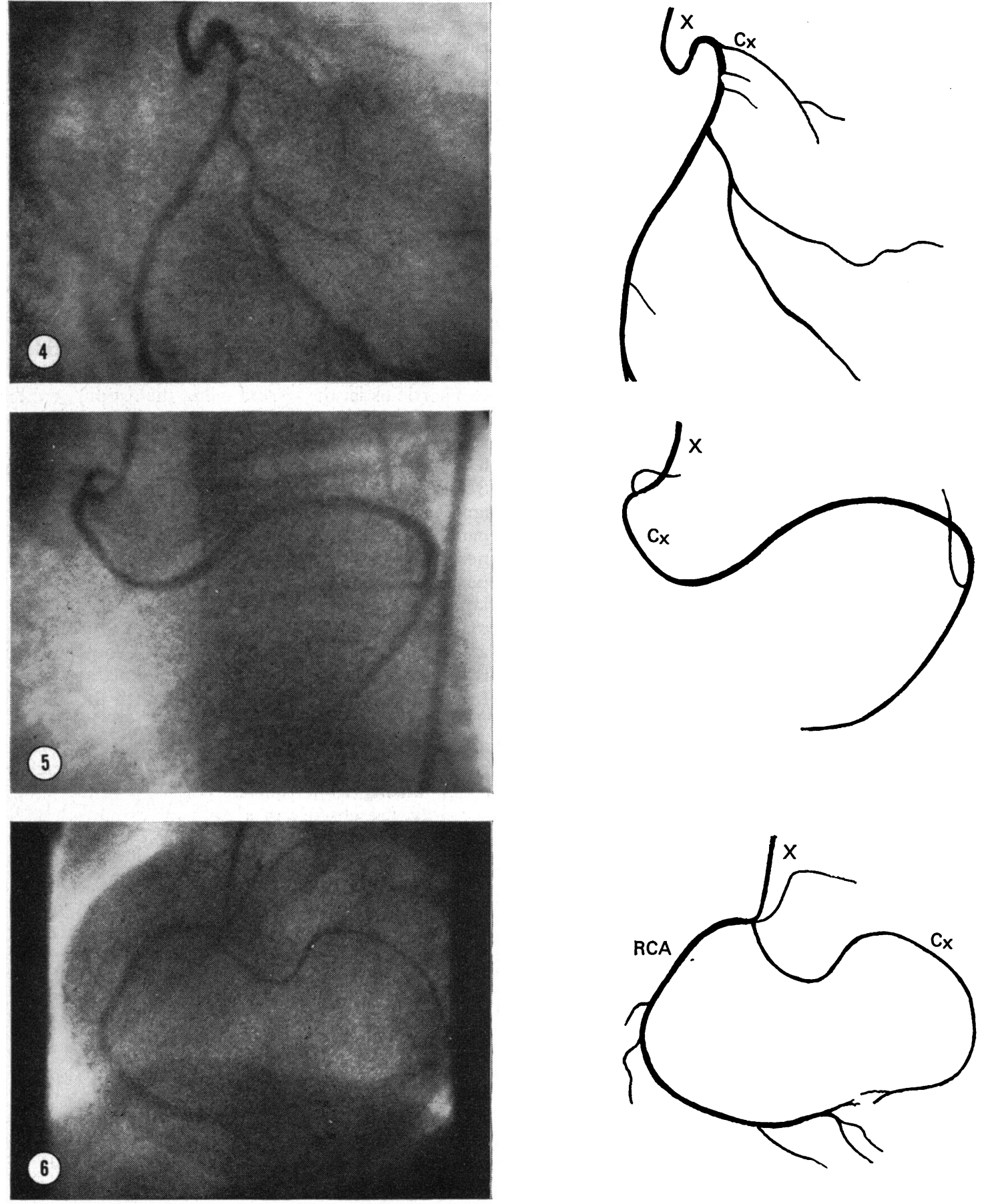


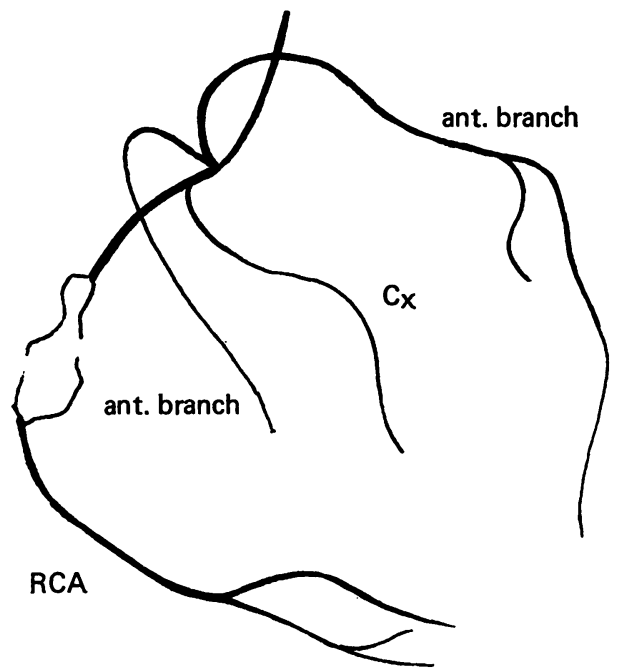

FIG. 7 (Case 5). Diagram of coronary arteriogram in the posteroanterior projection showing a single coronary artery. Two large branches pass anteriorly across the right ventricle to supply the territory of the left anterior descending artery. There is a single circumflex artery $(C X)$ passing behind the aorta towards the atrioventricular groove. The main right coronary artery $(R C A)$ is occluded and fills distally via collaterals.

James (196I) and Zumbo et al. (1965) have described a separate origin of the circumflex and anterior descending branches in $I$ per cent of patients. Rare variations in the origin of the left anterior descending artery have been demonstrated by Robicsek et al. (1967) and McNamara and Gross (1969). A single coronary artery may arise from the right coronary sinus, or the origin of either the circumflex or the anterior descending coronary artery may be from the right coronary artery itself or from a separate ostium in the right coronary sinus (James, I96I). This latter variation is encountered more commonly in association with other cardiac malformations such as tetralogy of Fallot or transposition of the great vessels.

The cases presented illustrate several variations of the normal anatomical pathway of the left circumflex coronary artery. In Case I there was no main left coronary artery, bifurcation of the main vessel being immediate. In this situation or with a very short main-stem, the catheter can easily be inserted too far into the left anterior descending artery and the circumflex vessel not visualized.

In Case 2 the left circumflex artery was only visualized after careful probing with the catheter in the left coronary sinus. In Cases 3 and 4 the abnormal vessel supplying the territory of the circumflex artery was found after probing in the right coronary sinus for a separate ostium. In Case 5 the whole of the blood supply of the heart arose from the right coronary sinus, with three abnormal vessels arising from the proximal right coronary artery. At operation, two of these branches ran across the front of the right ventricle to supply the territory of the left anterior descending artery; the third vessel passed from the right coronary artery along the posterior atrioventricular groove to the territory of the left circumflex artery.

These 5 cases illustrate anatomical variations which could easily lead to misdiagnosis. As these variations are not usually associated with physiological derangements, they have no typical clinical picture and are detected only by careful angiography. If the left circumflex artery is not visualized in its normal position one should first ensure that the catheter is not inserted too far into an early bifurcating left main artery. Both right and left coronary sinuses should then be carefully probed to exclude the presence of an anomalous vessel before assuming that the circumflex artery is either absent or totally occluded.

\section{References}

Edwards, J. E. (1958). Anomalous coronary arteries with special reference to arteriovenous-like communications. Circulation, 17, I001.

James, T. N. (1961). Anatomy of the Coronary Arteries. P. B. Hoeber, New York.

FIG. 4 (Case 2). Right anterior oblique projection showing the left circumflex artery (CX) arising directly from the aorta. Note the proximal stenosis.

FI G. 5 (Case 3). Posteroanterior projection showing that the circumflex artery $(C X)$ arises from the right coronary sinus and passes around the aortic root to enter the atrioventricular groove to supply branches to the left side of the heart. The left anterior descending artery was shown to be normal.

FI G. 6 (Case 4). Initial injection showed a normal right coronary artery. Slight withdrawal of the catheter tip enables both the right coronary artery $(R C A)$ and the circumflex artery $(C X)$ to be opacified. The left anterior descending artery was shown to be normal. 
McNamara, J. J., and Gross, R. E. (1969). Congenital coronary artery fistula. Surgery, 65, 59.

Ogden, J. A., and Goodyer, A. V. N. (1970). Patterns of distribution of the single coronary artery. Yale fournal of Biology and Medicine, 43, $1 \mathrm{I}$.

Ogden, J. A., and Stansel, H. C. (197I). Roentgenographic manifestations of congenital coronary artery disease. American fournal of Roentgenology, 113, 538.

Robicsek, F., Sanger, P. W., Daugherty, H. K, and Gallucci, V. (I967). Origin of the anterior interventricular (descending) coronary artery and vein from the left mammary vessels. Fournal of Thoracic and Cardiovascular Surgery, 53, 602.
Schlesinger, M. J., Zoll, P. M., and Wessler, S. (1949). The conus artery; a third coronary artery. American Heart fournal, 38, 823 .

Sewell, W. H. (r966). Roentgenographic anatomy of human coronary arteries. American fournal of Roentgenology, 97, 359.

Zumbo, O., Fani, K., Jarmolych, J., and Daoud, A. (1965). Coronary atherosclerosis and myocardial infarction in hearts with anomalous coronary arteries. Laboratory Investigation, 14, 571 .

Requests for reprints to Dr. P. R. Roy, National Heart Hospital, Westmoreland Street, London WI. 